\title{
34
}

\section{Sample-path Analysis of Queueing Systems with Leaky Bucket}

Shoji Kasahara $\dagger, \ddagger$

$\dagger$ Nara Institute of Science and Technology; Graduate School of Information Science

8916-5 Takayama, Ikoma Nara 630-01, Japan, Phone:+81-743-725353, Fax:+81-743-72-5359, E-mail: kasahara@is.aist-nara.ac.jp

$\ddagger$ Telecommunications Advancement Organization of Japan(TAO)

Shiba 2-31-19, Minato-Ku, Tokyo, 105 Japan

Toshiharu Hasegawa

Kyoto University; Department of Applied Systems Science, Graduate School of Engineering

Kyoto 606-01, Japan, E-mail: hasegawa@kuamp.kyoto-u.ac.jp

\begin{abstract}
This paper considers the queueing systems with leaky bucket using the samplepath argument. We consider two queueing models: one is the queueing system with leaky bucket in isolation and the other is that with multiple sources where each input is regulated by leaky bucket. In the former case, we show the sufficient condition for the rate stability of the data buffer process in terms of the token buffer process. In the latter case, supposing the burstiness constraints for the multiple inputs and the output, we give upper bounds of the buffer content process and the period during which the buffer content process is greater than a certain fixed point.
\end{abstract}

\section{Keywords}

Sample-path analysis, leaky bucket, rate stability 


\section{INTRODUCTION}

In the ATM-based Broadband ISDN (B-ISDN), it is expected that the different classes of traffic such as voice, video and data are multiplexed and carried to their destinations keeping their quality of service (QoS) satisfied the required level. In terms of the guarantee of QoS, the admission control scheme based on leaky bucket has been extensively studied and analyzed. One of the pioneering works is (Elwalid et al. 1991). In (Elwalid et al. 1991), the system with the access regulator has been analyzed in the context of the fluid queueing model.

In (Kulkarni et al. 1996), it turned out that the token buffer size doesn't play any role for the output process of the regulator using the leaky bucket. Our primary motivation of this paper is to investigate the relation between the token buffer size and the stability of the data buffer, and furthermore, the relation between the size of the token buffer and the behavior of the multiplexer's buffer where multiple regulated inputs are aggregated.

The queueing systems with leaky bucket have been studied assuming that the system is in equilibrium. In this context, it is not possible to capture the effects of the token buffer because it controls the bursty input from the source in the short time scale. Recently, (Cruz 1991a, Cruz 1991b) has proposed the concept of burstiness constraints. Using this constraints, we can derive the bounds of the queue length and the waiting time in the sample-path argument.

In this paper, we consider the queueing systems with leaky bucket using the sample-path argument. We consider the two queueing models: one is the queueing system with leaky bucket in isolation and the other is that with multiple sources where each input to the system is regulated by leaky bucket.

The sample-path analysis has played an increasingly important role in studying the limiting behavior of the queueing systems (see (Stidham et al. 1995) and the references therein). Sample-path techniques for queueing theory has been developed in (El-Taha et al. 1993, El-Taha et al. 1994, Stidham et al. 1993, Stidham et al. 1995). In (El-Taha et al. 1993, Stidham et al. 1993), the sample-path stability of an input-output process have been studied. In particular, a general sufficient condition for sample-path stability of an inputoutput system has been given. (El-Taha et al. 1994) analyzed the multi-server queueing system and derived some stability conditions. (Stidham et al. 1995) reviews variety kinds of application of sample-path analysis and showed how they all can be derived from the sample-path version of the renewal-reward theorem $(Y=\lambda X)$.

On the other hand, Chang (Chang 1994) analyzed the queueing networks in sample-path and probabilistic arguments. In the probabilistic argument, he developed the concept of the effective bandwidth. (Altman et al. 1993) also considered the bounds of the performance measures like a queue length and a cycle time from both sample-path and probabilistic arguments. 
Throughout this paper, we make no stochastic assumptions. The quantities and processes defined are deterministic. They may be thought of as representing a fixed sample path of a stochastic system, defined on some probability space.

The paper is organized as follows. In Section 2, we summarize the definition and some results of the stability of the general input-output process from (Stidham et al. 1995). In Section 3, we consider the sufficient condition of the rate stability for the queueing system with leaky bucket. In Section 4, we consider the boundaries of the fundamental quantities for the queueing system with multiple sources in which each generated input is regulated by leaky bucket.

\section{DEFINITIONS AND CHARACTERIZATION OF RATE STABILITY}

In this section, we summarize the definition and some results of the stability of an input-output process (Stidham et al. 1995).

Suppose that the state space $S=R^{+}$with the Borel $\sigma$-field $\mathcal{B}\left(R^{+}\right)$of subsets generated by the open sets. We consider an input-output process $\{Z(t), t \geq 0\}$ in which $Z(t)$ represents quantity in a system. We assume that $\{Z(t), t \geq 0\}$ is right continuous with left-hand limits. Let $A(t)(D(t))$ denote the cumulative input (output) to the system in $[0, t]$. Suppose that $\{A(t), t \geq 0\}$ and $\{D(t), t \geq 0\}$ are both nondecreasing, right-continuous processes. Then, $Z(t)$ is described as

$Z(t)=Z(0)+A(t)-D(t), \quad t \geq 0$

Note that $Z(t)$ has bounded variation on finite $t$-intervals.

Definition 1 An input-output process $\{Z(t), t \geq 0\}$ is said to be rate stable if

$\lim _{t \rightarrow \infty} t^{-1} Z(t)=0$

Then, the following lemma is immediate from (2).

Lemma 2 Suppose $t^{-1} A(t) \rightarrow \alpha<\infty$ as $t \rightarrow \infty$. Then the input-output process $\{Z(t), t \geq 0\}$ is stable if and only if $t^{-1} D(t) \rightarrow \alpha<\infty$ as $t \rightarrow \infty$.

Thus, an input-output process is rate stable if the long-run input and output rate exist and are finite and equal.

The following theorem gives sufficient conditions for rate stability of the input-output process $\{Z(t), t \geq 0\}$. 
Theorem 3 Consider the input-output process $\{Z(t), t \geq 0\}$ defined by (1). Suppose

$\lim _{t \rightarrow \infty} t^{-1} A(t)=\alpha$

and there exists a real number $z_{0}$ such that

$\lim _{t \rightarrow \infty} \frac{\int_{0}^{t} 1\left\{Z(s)>z_{0}\right\} d D(s)}{\int_{0}^{t} 1\left\{Z(s)>z_{0}\right\} d s}=\delta$

where $0<\alpha<\delta$. Then the process $\{Z(t), t \geq 0\}$ is rate-stable.

Proof. See (Stidham et al. 1995).

Corollary 4 Suppose the conditions (3) and (4) of Theorem 3 are satisfied with $z_{0}=0$, and $0<\alpha<\delta$. Suppose also that

$\lim _{t \rightarrow \infty} t^{-1} \int_{0}^{t} 1\{Z(s)=0\} d D(s)=0$.

Then $p(0):=\lim _{t \rightarrow \infty} t^{-1} \int_{0}^{t} 1\{Z(s)=0\} d s$ is well-defined and

$p(0)=1-\rho$,

where $\rho=\alpha / \delta$.

Proof. See (Stidham et al. 1995).

\section{QUEUEING SYSTEM WITH LEAKY BUCKET}

In this section, we consider the rate stability of the queueing system with leaky bucket. We show our queueing model in Figure 1. 


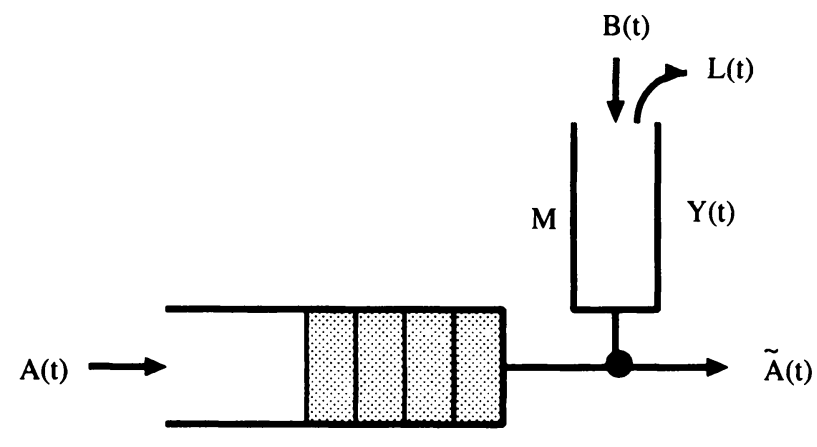

$\mathrm{Z}(\mathrm{t})$

Figure 1 Queueing System with Leaky Bucket

The system has an infinite data buffer for messages and the finite token buffer whose size is $M$. Tokens arrive at the token buffer and arriving tokens which find the token buffer full are lost.

Arriving message is sent to the output and a token is removed from the token buffer if there is at least one token in the buffer. If there are no tokens in the token buffer, the message waits for the token arriving and is sent to the output when the token arrives.

In this system, we define the following processes;

$A(t)$ : cumulative number of arriving messages at time $t$,

$B(t)$ : cumulative number of arriving tokens at $t$,

$\tilde{A}(t)$ : cumulative number of messages departing from the system at $t$,

$L(t)$ : cumulative number of lost tokens at $t$.

Note that the output process of the token buffer is equal to $\tilde{A}(t)$.

Let $\{Y(t): 0 \leq Y(t) \leq M, t \geq 0\}$ denote the token buffer process at time $t$. Then $Y(t)$ satisfies the following equation

$Y(t)=Y(0)+B(t)-\tilde{A}(t)-L(t)$

Using (7), (1) becomes

$Z(t)=Z(0)+A(t)-Y(0)-B(t)+Y(t)+L(t)$.

First, we give a preliminary result that is of independent interest.

Lemma 5 Consider the input-output process $\{Z(t), t \geq 0\}$ defined by (8). Let $\alpha$ and $\gamma$ be non-negative constants. Suppose 
1. the input process of the data buffer satisfies

$$
\lim _{t \rightarrow \infty} t^{-1} A(t)=\alpha
$$

2. the input process of the token buffer satisfies

$$
\lim _{t \rightarrow \infty} t^{-1} B(t)=\gamma
$$

where $0<\alpha<\gamma<\infty$.

Then the event $\{Y(t)>0\}$ occurs infinitely often as $t \rightarrow \infty$. That is, for every $t_{0} \geq 0$, there exists a $t \geq t_{0}$ such that $Y(t)>0$.

Proof. Without the loss of generality, we assume that $Z(0)=Y(0)=0$, i.e.,

$$
Z(t)=A(t)-B(t)+Y(t)+L(t)
$$

The proof is by contradiction. It follows from (9) and (10) that, for every $\epsilon>0$, there exists a $T$ such that, for all $t \geq T$,

$$
\begin{aligned}
& (\alpha-\epsilon) t \leq A(t) \leq(\alpha+\epsilon) t, \\
& (\gamma-\epsilon) t \leq B(t) \leq(\gamma+\epsilon) t,
\end{aligned}
$$

Now suppose $0<\epsilon<(\gamma-\alpha) / 2$. Suppose that the event $\{Y(t)>0\}$ does not occur infinitely often as $t \rightarrow \infty$. Then there exists a $t_{0}$ such that, for all $t \geq t_{0}, Y(t)=0$. Hence, using (11), we have for all $t \geq t_{0}$

$$
\begin{aligned}
Z(t) & =Z\left(t_{0}\right)+\left\{A(t)-A\left(t_{0}\right)\right\}-\left\{B(t)-B\left(t_{0}\right)\right\}+\left\{L(t)-L\left(t_{0}\right)\right\} \\
& =Z\left(t_{0}\right)-A\left(t_{0}\right)+B\left(t_{0}\right)+A(t)-B(t) \\
& \leq Z\left(t_{0}\right)-A\left(t_{0}\right)+(\alpha-\gamma+2 \epsilon) t
\end{aligned}
$$

where the second equality follows from the fact that there are no arrival tokens lost after $Y(t)=0$. Since $(\alpha-\gamma+2 \epsilon)<0$, by choosing $t$ sufficiently large, we can make the quantity on the right-hand side of the last equality negative, thus leading to a contradiction of $Z(t) \geq 0$. Therefore, for every $t_{0} \geq T$, there exists at least one $t>t_{0}$ such that $Y(t)>0$. That is, the event $\{Y(t)>0\}$ occurs infinitely often as $t \rightarrow \infty$.

Now we give the following theorem for the sufficient condition of the rate stability of $Z(t)$ based on $Y(t)$. 
1. $A(t)$ satisfies

$$
\lim _{t \rightarrow \infty} t^{-1} A(t)=\alpha
$$

2. there exists a real number $c$ such that $0<c \leq M$ and

$$
\lim _{t \rightarrow \infty} \frac{\int_{0}^{t} 1\{Y(s) \leq c\} d B(s)}{\int_{0}^{t} 1\{Y(s) \leq c\} d s}=\gamma^{\prime}
$$

where $0<\alpha<\gamma^{\prime}$;

3. The event $\{Y(t)>c\}$ occurs infinitely often as $t \rightarrow \infty$.

Then the process $\{Z(t), t \geq 0\}$ is rate stable.

Proof. The proof is by contradiction and is similar to those of Theorem 5.1. of (El-Taha et al. 1993), Theorem 2.2. of (Stidham et al. 1993) and Theorem 2.2. of (El-Taha et al. 1994).

Suppose that $\{Z(t), t \geq 0\}$ is not rate stable. Then there exists a $\xi>0$ and an increasing sequence of time points $\left\{\tau_{n}, n \geq 1\right\}$, with $\tau_{n} \rightarrow \infty$ as $n \rightarrow \infty$, such that $Z\left(\tau_{n}\right) \geq \xi \tau_{n}$ for all $n \geq 1$. Without the loss of generality, we consider the process defined in (11). We define $U(t)$ as

$$
U(t)=\int_{0}^{t} 1\{Y(s) \leq c\} d s .
$$

It follows from (14) and (15) that, for every $\epsilon>0$, there exists a $T<\infty$ such that

$$
\begin{aligned}
& (\alpha-\epsilon) t \leq A(t) \leq(\alpha+\epsilon) t, \quad t \geq T \\
& \left(\gamma^{\prime}-\epsilon\right) U(t) \leq \int_{0}^{t} 1\{Y(s) \leq c\} d B(s) \leq\left(\gamma^{\prime}+\epsilon\right) U(t), \quad t \geq T .
\end{aligned}
$$

Note that from the assumption 3., $U(t) \rightarrow \infty$ as $t \rightarrow \infty$.

Let $a_{n}=\sup \left\{s: s<\tau_{n}, Y(s)>c\right\}$. Then, it follows that

$$
U\left(\tau_{n}\right)=U\left(a_{n}\right)+\tau_{n}-a_{n}
$$

Moreover, the assumption 3. show that $a_{n} \rightarrow \infty$ as $n \rightarrow \infty$. Now we choose $\epsilon<\left(\xi-c / a_{n}\right) / 5$. We have $a_{n}>T$ for sufficiently large $n$. For such $n$, it follows from (16), (17) and (18) that

$$
Z\left(a_{n}\right)=Z\left(\tau_{n}\right)-\left(A\left(\tau_{n}\right)-A\left(a_{n}\right)\right)+\left(B\left(\tau_{n}\right)-B\left(a_{n}\right)\right)
$$




$$
\begin{aligned}
&-\left(Y\left(\tau_{n}\right)-Y\left(a_{n}\right)\right)-\left(L\left(\tau_{n}\right)-L\left(a_{n}\right)\right) \\
&= Z\left(\tau_{n}\right)-\left(A\left(\tau_{n}\right)-A\left(a_{n}\right)\right)+\left(B\left(\tau_{n}\right)-B\left(a_{n}\right)\right)-\left(Y\left(\tau_{n}\right)-Y\left(a_{n}\right)\right) \\
& \geq \xi \tau_{n}-(\alpha+\epsilon) \tau_{n}+(\alpha-\epsilon) a_{n}+\left(\gamma^{\prime}-\epsilon\right) U\left(\tau_{n}\right)-\left(\gamma^{\prime}+\epsilon\right) U\left(a_{n}\right)-c \\
&= \xi \tau_{n}+\left(\gamma^{\prime}-\alpha-2 \epsilon\right) \tau_{n}+\left(\alpha-\gamma^{\prime}\right) a_{n}-2 \epsilon U\left(a_{n}\right)-c \\
&= \xi \tau_{n}-2 \epsilon a_{n}+\left(\gamma^{\prime}-\alpha-2 \epsilon\right)\left(\tau_{n}-a_{n}\right)-2 \epsilon U\left(a_{n}\right)-c \\
& \geq \xi \tau_{n}-2 \epsilon a_{n}-2 \epsilon U\left(a_{n}\right)-c \\
& \geq \xi a_{n}-2 \epsilon a_{n}-2 \epsilon a_{n}-c \\
&=(\xi-4 \epsilon) a_{n}-c \\
&>\epsilon a_{n},
\end{aligned}
$$

where the second equality is obtained from the fact that there are no lost tokens for $a_{n}<t<\tau_{n}$. On the other hand, $Z\left(a_{n}\right)=0$ when $Y\left(a_{n}\right)>c$ for all $n$. Thus we have a contradiction and the proof is complete.

Remark 7 Since $\epsilon$ is arbitrarily chosen from the positive real number, we can choose its value less than $\left(\xi-c / a_{n}\right) / 5 .\left\{a_{n}, n \geq 1\right\}$ is the increasing sequence as $n$ is getting large and hence the effect of $c / a_{n}$ becomes small and negligible. This means that the effect of the token buffer size becomes negligible if we consider the stability of the system in the long time scale.

If we restrict the condition 2 . of Theorem 6 to the event $\{Y(t)=0\}$, we obtain the following corollary.

Corollary 8 Suppose the condition 1. of Theorem 6 is satisfied. Suppose also that

$\lim _{t \rightarrow \infty} \frac{\int_{0}^{t} 1\{Y(s)=0\} d B(s)}{\int_{0}^{t} 1\{Y(s)=0\} d s}=\gamma^{\prime}$

where $0<\alpha<\gamma^{\prime}$. Then the process $Z(t)$ is rate stable.

Proof. The condition (19) is equivalent to the following condition

$$
\lim _{t \rightarrow \infty} \frac{\int_{0}^{t} 1\{Z(s)>0\} d B(s)}{\int_{0}^{t} 1\{Z(s)>0\} d s}=\gamma^{\prime}
$$

Then it is immediate from Theorem 3. 


\section{QUEUEING SYSTEM WITH REGULATED INPUTS}

In this section, we consider the queueing system with the regulated inputs (see Figure 2). This system is a typical model of the ATM multiplexer. Here, we assume that there are $K$ sources and that each source generates the regulated input traffic using the leaky bucket scheme considered in section 4 .

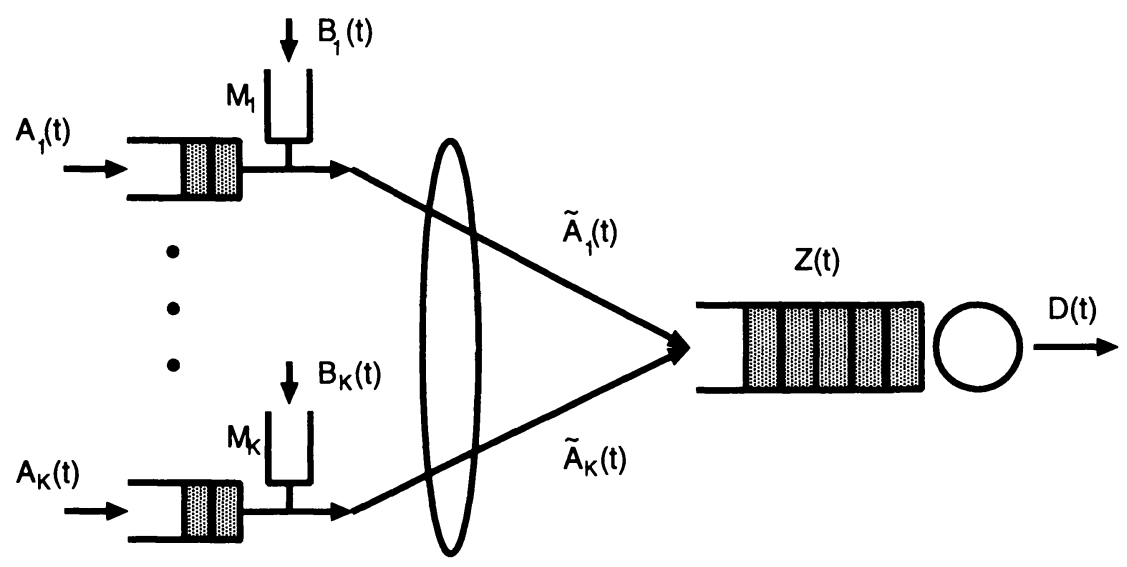

Figure 2 Queueing System with Regulated Inputs

Sources are labeled $i=1, \cdots, K$ in sequence. The size of the token buffer of $i$ th source is $M_{i}$. In similar to the previous section, we use the following notations:

$A_{i}(t)$ : cumulative number of messages arriving to the $i$ th regulator at $t$, $B_{i}(t)$ : cumulative number of arriving tokens of the $i$ th regulator at $t$, $\tilde{A}_{i}(t)$ : cumulative number of messages departing from $i$ th regulator at $t$.

We also define $\lim _{t \rightarrow \infty} A_{i}(t) / t=\alpha_{i}$ and $\lim _{t \rightarrow \infty} B_{i}(t) / t=\gamma_{i}$ for $i=1, \cdots, K$. We assume that $\alpha_{i}<\gamma_{i}$ for $i=1, \cdots, K$. Clearly, the message buffer process of each source is stable.

$K$ regulated inputs are aggregated and sent to the shared buffer. Let $Z(t)$ denote the buffer contents of the system at time $t$. We define $D(t)$ as the total amount of the output process at $t$.

In this system, the total amount of inputs, denoted by $\tilde{A}(t)$, is equal to $\sum_{i=1}^{K} \tilde{A}_{i}(t)$. Let $\gamma$ denote the average rate of the total input. Then, we have

$\lim _{t \rightarrow \infty} \frac{\tilde{A}(t)}{t} \leq \lim _{t \rightarrow \infty} \frac{\sum_{i=1}^{K} B_{i}(t)}{t}=\sum_{i=1}^{K} \gamma_{i} \equiv \gamma$. 
Now we assume that there exists a real number $z_{0}$ such that

$\lim _{t \rightarrow \infty} \frac{\int_{0}^{t} 1\left\{Z(s)>z_{0}\right\} d D(s)}{\int_{0}^{t} 1\left\{Z(s)>z_{0}\right\} d s}=\delta$

where $\gamma<\delta$. Then, from Theorem $3,\{Z(t), t \geq 0\}$ is rate stable.

Following to (Altman et al. 1993), we define the "burstiness" of arrival and departure processes during a time interval $[s, t)$ by

$B_{s, t}^{\tilde{A}}=\tilde{A}(t)-\tilde{A}(s)-\gamma(t-s)$,

$B_{s, t}^{D}=D(t)-D(s)-\delta \int_{s}^{t} 1\left\{Z(\tau)>z_{0}\right\} d \tau$

That is, $B_{s, t}^{\bar{A}}$ is the difference between the actual input and the input constantly generated with the average rate $\gamma$ throughout the interval $[s, t)$. Similarly, $B_{s, t}^{D}$ is the difference between the actual output and the output constantly served with the average rate $\delta$ when the queue length is greater than $z_{0}$.

Let $b_{0}=0$ and for $n=1,2, \cdots$, define the following time points

$b_{n}=\inf \left\{t>b_{n-1}: Z(t-) \leq z_{0}, Z(t)>z_{0}\right\}$,

$e_{n}=\inf \left\{t>b_{n}: Z(t-)>z_{0}, Z(t) \leq z_{0}\right\}$

In other words, the $n$th event of $\left\{Z(t)>z_{0}\right\}$ starts at time $b_{n}$ and ends at $e_{n}$. Let $T_{n}:=e_{n}-b_{n}$.

Now we show that supposing the Cruz bounds for the burstiness of both arrival and departure processes, $T_{n}$ and $Z(t)$ are bounded for $n \geq 1$ and $b_{n} \leq t \leq e_{n}$. Specifically, we assume that

$\tilde{A}(t)-\tilde{A}(s) \leq \gamma(t-s)+M, 0 \leq s<t$,

$D(t)-D(s) \geq \delta \int_{s}^{t} 1\left\{Z(\tau)>z_{0}\right\} d \tau-\sigma_{D}, 0 \leq s<t$,

where $M=\sum_{i=1}^{K} M_{i}$ and $\sigma_{D}$ is non-negative constant. In (Altman et al. 1993), (23) and (24) are referred to as linear burstiness bounds. It follows from (21), (22), (23) and (24) that

$B_{s, t}^{\tilde{A}} \leq M, \quad-B_{s, t}^{D} \leq \sigma_{D}, \quad 0 \leq s<t$. 
Remark 9 From (23), for $\epsilon>0$, the worst case of the arrival process during $[t, t+\epsilon)$ is

$\tilde{A}(t+\epsilon) \leq \tilde{A}(t)+M, 0 \leq t$.

This means that the total amount of token buffers bounds the maximum amount of the bursty input. After the worst case occurs, the input rate is bounded by $\gamma$, the total amount of token generation rates.

Theorem 10 For $n \geq 1$ and $b_{n} \leq t \leq e_{n}$,

$T_{n} \leq \frac{z_{0}+M+\sigma_{D}}{\delta-\gamma}$,

$Z(t) \leq z_{0}+M+\rho \sigma_{D}$

where $\rho=\gamma / \delta$.

Proof. First, we show (26). It follows from (22) that

$$
\begin{aligned}
\delta T_{n} & =D\left(e_{n}\right)-D\left(b_{n}\right)-B_{b_{n}, e_{n}}^{D} \\
& \leq z_{0}+\gamma T_{n}+B_{b_{n}, e_{n}}^{\tilde{A}}-B_{b_{n}, e_{n}}^{D} \\
& \leq z_{0}+\gamma T_{n}+M+\sigma_{D},
\end{aligned}
$$

since the output during $T_{n}$ can be no more than $z_{0}$, the work at $b_{n}$, plus the input during $T_{n}$ and second inequality follows from (25).

To show (27), note that for $b_{n} \leq t \leq e_{n}$,

$D(t)-D\left(b_{n}\right)=\delta\left(t-b_{n}\right)+B_{b_{n}, t}^{D}$.

Then, we obtain

$$
\begin{aligned}
Z(t) & =Z\left(b_{n}\right)+\alpha\left(t-b_{n}\right)+B_{t_{n}, t}^{\tilde{A}}-\left\{D(t)-D\left(b_{n}\right)\right\} \\
& =z_{0}+\rho\left\{D(t)-D\left(b_{n}\right)-B_{b_{n}, t}^{D}\right\}+B_{t_{n}, t}^{\tilde{A}}-\left\{D(t)-D\left(b_{n}\right)\right\} \\
& =z_{0}+B_{t_{n}, t}^{\tilde{A}}-\rho B_{b_{n}, t}^{D}-(1-\rho)\left\{D(t)-D\left(b_{n}\right)\right\} \\
& \leq z_{0}+B_{t_{n}, t}^{\tilde{A}}-\rho B_{b_{n}, t}^{D} \\
& \leq z_{0}+M+\rho \sigma_{D} .
\end{aligned}
$$

Remark 11 As for the ATM multiplexer, the arriving cell is processed with 
the constant rate. In this case, there are no burstiness in terms of the output process $D(t)$ and hence $\sigma_{D}=0$. If the condition (20) holds at $z_{0}=0, Z(t)$ is bounded by $M$. That is, if the total input rate is smaller than the output rate, the queue length of the multiplexer is no more greater than the total amount of token buffers.

\section{REFERENCES}

Altman, E., Foss, S.G., Riehl, E.R. and Stidham, Jr., S. (1993) Performance bounds and pathwise stability for generalized vacation and polling systems. Technical Report No. 93-8, Dept. of Operations Research, University of North Carolina, Chapel Hill, NC 27599.

Chang, C.S. (1994) Stability, queue length, and delay of deterministic and stochastic queueing networks. IEEE Trans. Automa. Contr., 39(5), 913931.

Cruz, R.L. (1991) A calculus for network delay, part I: network elements in isolation. IEEE Trans. Inform. Theory., 37(1), 114-131.

Cruz, R.L. (1991) A calculus for network delay, part II: network analysis. IEEE Trans. Inform. Theory., 37(1), 132-141,

El-Taha, M. and Stidham, Jr., S. (1993) Sample-path analysis of stochastic discrete-event systems. J. Discrete Event Dynamic Systems, 3, 325-346.

El-Taha, M. and Stidham, Jr., S. Sample-path conditions for multiserver input-output processes. J. Appl. Math. Stoch. Anal., 7, 437-456.

Elwalid, A.I. and Mitra, D. (1991) Analysis and design of rate-based congestion control of high speed networks, I: stochastic fluid models, access regulation. Queueing Systems, 9, 29-64.

Kulkarni, V.G. and Gautam, N. (1996) Leaky buckets: sizing and admission control. Technical Report No. 95-10, Dept. of Operations Research, University of North Carolina, Chapel Hill, NC 27599.

Stidham, Jr., S. and El-Taha, M. (1993) A note on sample-path stability conditions for input-output processes. Operations Research Letters, 14, $1-7$.

Stidham, Jr., S. and El-Taha, M. (1995) Sample-path techniques in queueing theory. Advances in Queueing (ed. J.H. Dshalalow), CRC Press, Inc., 119-166. 